\title{
Experimental Details*
}

*Blocks are designated by P2VP for poly-2-vinylpyridine and PS for polystyrene. Molecular weights of the individual blocks are given after the block notation in units of daltons.

Solution Preparation. P2VP50000, PS7800-P2VP10000, PS13800-P2VP47000, and PS60100-P2VP46900 were purchased from Polymer Source (Dorval, Canada) and had polydispersity indices less than 1.11. PS29100 was purchased from Aldrich (St. Louis, MO) and had a polydispersity index of 1.04. Block copolymers and PS were dissolved in filtered toluene (J.T. Baker, Aston, PA) while P2VP was dissolved in THF (EM Science). All solutions were approximately $2 \mathrm{~g} / \mathrm{L}$.

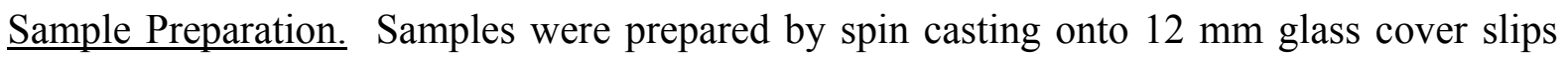
from Fisher Scientific (Pittsburgh, PA) at $1000 \mathrm{rpm}$ for 2 minutes using a Headway Research ED101D photo resist spinner.

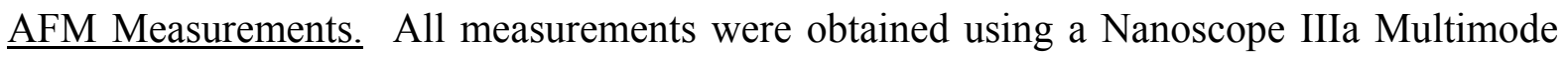
from Digital Instruments (Santa Barbara, CA). Spring constants were calibrated using a Park Scientific Instruments (Sunnyvale, CA) force constant calibration cantilever and were approximately $0.07 \mathrm{~N} / \mathrm{m}$ for all cantilevers except the cantilever used on P2VP $(0.03 \mathrm{~N} / \mathrm{m})$. All measurements were done in $0.45 \mu \mathrm{m}$ filtered $10 \mathrm{mM}$ sodium acetate buffer (EM Science) with the water purified to $18 \mathrm{M} \Omega \cdot \mathrm{cm}$ resistivity (Barnstead NANO pure filter). Force volumes of $16 \mathrm{x}$ 16 x 512 points were collected using Nanoscope IIIa software. Tip contamination for the data presented was irrelevant since the frequency of force plots displaying elastic responses significantly increases upon tip contamination. Therefore, the single rupture data presented does not represent polymer-substrate detachment but rather tip-polymer detachment. 
Data Analysis. The loading rate was obtained directly from our force plots using Equation S1.

$$
r_{\text {rupture }}=\frac{\Delta F \cdot S_{\text {rate }}}{n+1}
$$

Here, $\Delta \mathrm{F}$ is the change in the attractive force from the point of rupture to four points prior to this rupture, $\mathrm{n}$ is 1 less than the number of points used to obtain $\Delta \mathrm{F}$, and $\mathrm{S}_{\text {rate }}$ is the sampling rate in the data acquisition in points/s. The velocity for the Hummer plots is then calculated using the loading rate and spring constant of the system which incorporates the spring constants of both the cantilever and the extended polymer chain. Bins for the force $v s$. velocity plots were created to obtain approximately equal numbers of points per bin.

\section{Theoretical Model For Analyzing Molecular Pulling Experiments}

To investigate the mechanically induced extension of PS, P2VP, and their block copolymers, this study relied on a model developed by Hummer and Szabo (Hummer, G.; Szabo, A. Biophys. $J ., 2003,85,5)$, hereafter referred to as the Hummer model. This model improves upon the model established by Bell and Evans since the position of the rate-limiting barrier is allowed to fluctuate under the pulling potential and an activation prefactor of $\mathrm{k}_{\mathrm{B}} \mathrm{T} / \mathrm{h}$ is not assumed. Additional advantages of using this model include obtaining estimates of the polymer's spring constant and the system's interfacial stiffness. However, with more free parameters in the model, the initial inputs need to be more accurate making it slightly more difficult than the Bell/Evans model in terms of ease of implementation. Furthermore, with more parameters, too much uncertainty can result if few data points are available.

The Hummer model assumes a molecular free energy surface $\left(\mathrm{V}_{\mathrm{o}}(\mathrm{x})\right)$ whose potential of mean force is given by Equation S2.

$$
V(x, t)=V_{o}(x)+V_{s}(x-v t)
$$


Here, the reaction coordinate, $\mathrm{x}$, is coupled to the AFM's piezo velocity, v. Hummer also assumes the free energy surface is characterized by Equation S3

$$
B V_{o}(x)=\left\{\begin{array}{ll}
\frac{1}{2} k_{m} x^{2} & \left(x<x^{\ddagger}\right) \\
-\infty & \left(x \geq x^{\ddagger}\right)
\end{array}\right\}
$$

where $\mathrm{k}_{\mathrm{m}}$ represents the molecular spring constant, $\mathrm{B}^{-1}=\mathrm{k}_{\mathrm{B}} \mathrm{T}$, and $\mathrm{x}^{\ddagger}$ corresponds to the distance from the free energy minimum to the transition state projected along the direction of applied force. Since it is assumed that the system undergoes Brownian motion on the free energy surface, Kramers theory provides the relationship between the rate of rupture in the absence of pulling $\left(\mathrm{k}_{\mathrm{off}}(0)\right)$ and the system's properties through Equation S4.

$$
k_{o f f}(0)\left(x^{\ddagger} ; k_{m}\right) \approx(2 \pi)^{-1 / 2} D k_{m}^{3 / 2} x^{\ddagger} e^{-B \Delta G^{\ddagger}}, \quad B \Delta G^{\ddagger}=\frac{k_{m}\left(x^{\ddagger}\right)^{2}}{2}
$$

Here $\mathrm{D}$ and $\Delta \mathrm{G}^{\ddagger}$ represent the diffusion coefficient and the unfolding barrier height, respectively.

Hummer et al. show how to extract the rate constant from velocity dependent pulling experiments by analyzing the statistics of the force at rupture. For intermediate pulling velocities, a fit of the average rupture force $(\bar{F})$ vs. velocity (v) (Equation S5) allows the molecular spring constant $\left(\mathrm{k}_{\mathrm{m}}{ }^{*}=\mathrm{k}_{\mathrm{B}} T \mathrm{k}_{\mathrm{m}}\right)$, the barrier distance $\left(\mathrm{x}^{\sharp}\right)$, and the kinetic offrate $\left(\mathrm{k}_{\text {off }}(0)\right)$ in the absence of pulling to be obtained.

$$
B \bar{F}=k_{m} x^{\ddagger}-\left[2 k \ln \frac{k_{o f f}(0) e^{\gamma+k_{m}\left(x^{\ddagger}\right)^{2} / 2}}{k_{s} v x^{\ddagger}\left(k_{m} / k\right)^{3 / 2}}\right]^{1 / 2}, k=k_{m}+k_{s}
$$

Here $\mathrm{k}_{\mathrm{s}}$ is an effective spring constant that incorporates the spring constants of both the cantilever and the extended polymer chain, and $\gamma$ is the Euler-Mascheroni constant $(\gamma=$ $0.5772 \ldots)$. Since $\mathrm{k}_{\mathrm{s}}$ represents multiple springs in series, we can actually obtain the polymer's 
spring constant using Equation S6 where $\mathrm{k}_{\mathrm{s}}{ }^{*}=\mathrm{k}_{\mathrm{B}} \mathrm{Tk}_{\mathrm{s}}$, and $\mathrm{k}_{\mathrm{chain}}$ and $\mathrm{k}_{\mathrm{c}}$ represent the polymer and the cantilever's spring constant, respectively.

$$
\frac{1}{k_{s}^{*}}=\frac{1}{k_{\text {chain }}}+\frac{1}{k_{c}}
$$

In the data reported here, the velocity was determined directly from the force plots using the loading rate and spring constant of the system as discussed earlier; therefore, a factor of $\left(1+\frac{k_{c} k_{m}+k_{c} k_{c h a i n}}{k_{m} k_{c h a i n}}\right)$ was multiplied to $\mathrm{v}$ in Equation S5 to account for the tip's velocity. We note that by using the maximum loading rate, rather than considering the entire history of the force, the reported $k_{\text {off }}$, and $x^{\ddagger}$ are expected to represent lower bounds, and $\Delta \mathrm{G}^{\star}$ is expected to represent an upper bound.

\section{Results}

$\underline{\text { AFM Image of a Block Co-Polymer Surface. }}$

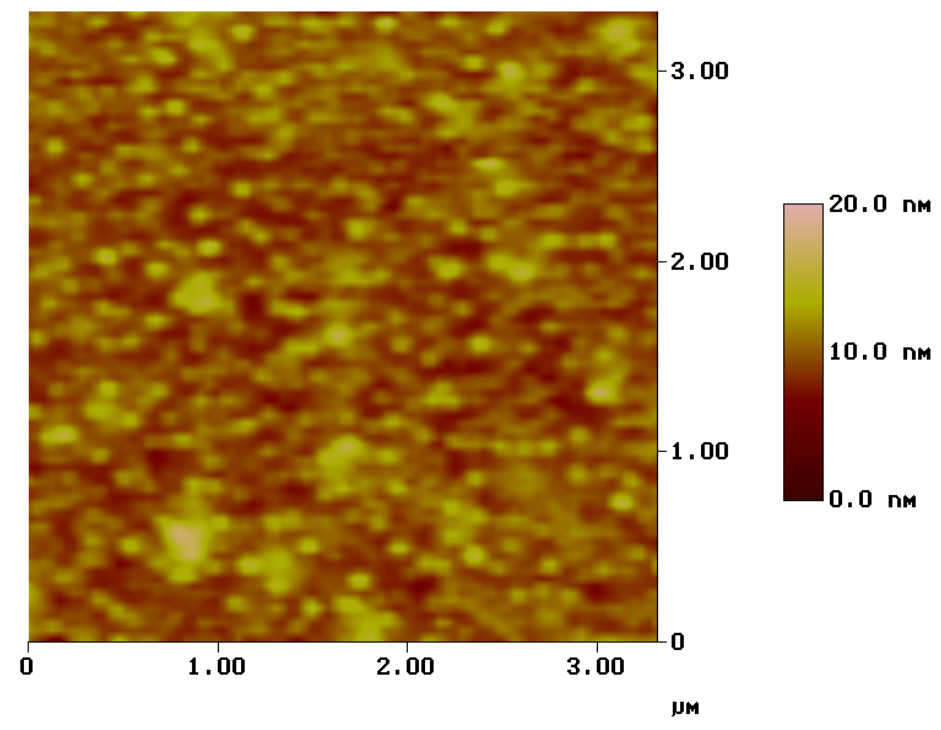

Figure S1: A height image of PS13800-P2VP47000 revealing structures that appear to be micellular in nature. Image is about 3.5 microns x 3.5 microns. 
Graphical Representation of $\mathrm{k}_{\text {chain }}$ as a function of P2VP Molecular Weight.

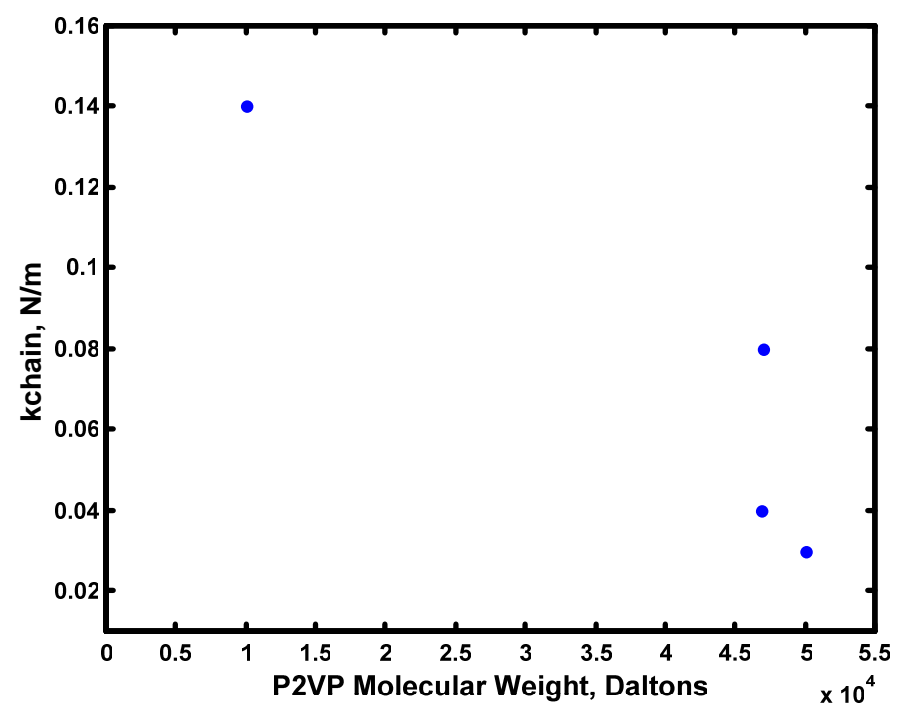

Figure S2: $k_{\text {chain }}$ as a function of P2VP molecular weight. 

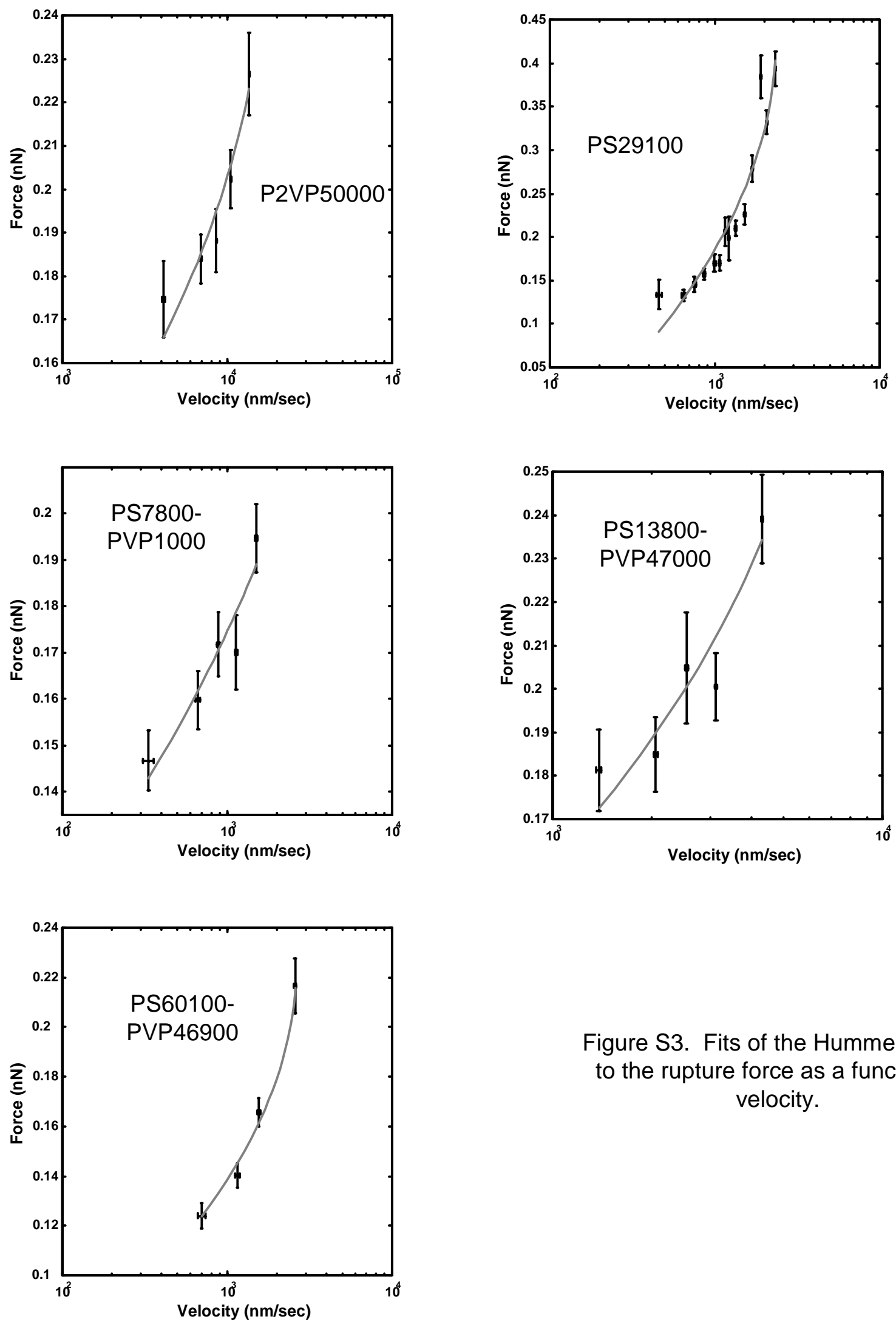

Figure S3. Fits of the Hummer model to the rupture force as a function of velocity. 